Article

\title{
Investigation of the Functional Decline in Proprioceptors for Low Back Pain Using the Sweep Frequency Method
}

\author{
Reiya Nishio ${ }^{1, * \mathbb{C}}$, Yohei Ito ${ }^{1}$, Yoshifumi Morita ${ }^{1}$, Tadashi Ito ${ }^{2} \mathbb{D}$, Kazunori Yamazaki $^{3}$ \\ and Yoshihito Sakai ${ }^{4}$ \\ 1 Department of Electrical and Mechanical Engineering, Graduate School of Engineering, Nagoya Institute of \\ Technology, Nagoya 466-8555, Japan; y.ito.359@nitech.jp (Y.I.); morita@nitech.ac.jp (Y.M.) \\ 2 Three-Dimensional Motion Analysis Room, Aichi Prefectural Mikawa Aoitori Medical and Rehabilitation \\ Center for Developmental Disabilities, Okazaki 444-0002, Japan; sanjigen@mikawa-aoitori.jp \\ 3 Faculty of Clinical Engineering, School of Medical Sciences, Fujita Health University, \\ Toyoake 470-1192, Japan; ymzkk@fujita-hu.ac.jp \\ 4 Department of Orthopedic Surgery, National Center for Geriatrics and Gerontology, Obu 474-8511, Japan; \\ jsakai@ncgg.go.jp \\ * Correspondence: r.nishio.115@nitech.jp; Tel.: +81-52-735-5412
}

Received: 24 October 2019; Accepted: 18 November 2019; Published: 20 November 2019

\begin{abstract}
The purpose of this study was to investigate the functional decline in proprioceptors in patients with low back pain (LBP) by evaluating the entire range of response frequencies of proprioceptors. In previous studies, the function of proprioceptors was only evaluated by single frequency vibrations. However, because it is assumed that individual differences exist in response frequencies of proprioceptors, we developed a method using vibration with sweep frequency covering the entire range of response frequencies of proprioceptors. The center of pressure was determined in 35 elderly patients with non-specific LBP (NSLBP) and 28 elderly individuals with non-LBP (NLBP) during upright stances on a balance board without vision. Local vibratory stimulations to lower leg or trunk muscles were continuously varied between 27 and $272 \mathrm{~Hz}$ over $60 \mathrm{~s}$ to evaluate the proprioceptive predominance of a body location using the relative proprioceptive weighting ratio (RPW). Compared with the NLBP group, the NSLBP group exhibited a lower RPW value for the Vater-Pacini corpuscles. Thus, the NSLBP group relied more on the input of Vater-Pacini corpuscles in the trunk. A reduction in lower leg sensitivity at the Vater-Pacini corpuscles in the NSLBP group was observed.
\end{abstract}

Keywords: vibratory stimulation device; local muscle vibration; proprioceptors; low back pain; response frequency; postural control; Vater-Pacini corpuscles

\section{Introduction}

Worldwide, low back pain (LBP) has become the main cause of disability [1]. However, its causes are unidentifiable in $85 \%$ of the cases [2]. Postural control impairment is considered one of the main causes of LBP [3]. Postural control is affected by sensory inputs from visual and vestibular senses and somatosensory receptors which provide superficial and proprioception. A previous study indicated that the feedback control on the trunk posture is mainly influenced by the visual and proprioception [4]. Also, another previous study suggested that the ability to select and reweigh sensory inputs to adapt to situations is a critical factor for postural control [5]. In addition, non-specific LBP (NSLBP) may alter postural control by refocusing proprioception from the trunk to the ankles [6]. Furthermore, reduced 
precision of trunk movement in NSLBP might be explained by proprioceptive deficits [7]. Therefore, NSLBP could be caused by a decline in proprioception leading to problems in postural control and LBP.

Proprioception in the lower leg and trunk muscle plays an important role in maintaining postural stability [8]. Each proprioceptor has a response frequency of vibration. Meissner corpuscles are sensitive to lower vibration frequencies (peaks around $30 \mathrm{~Hz}$ ), whereas the Vater-Pacini corpuscles are sensitive to higher frequencies (peaks around $240 \mathrm{~Hz}$ ) [9]. Muscle spindles have a response frequency range of $40-100 \mathrm{~Hz}$ [10]. Mechanical vibration is commonly used to test externally induced balance control. This method has been widely used to investigate the role of muscle spindle in research focusing on postural control [11-14]. However, the role of other proprioceptors in NSLBP has not been evaluated. Thus, comparing postural sway of NSLBP during various local frequency vibrations with that of non-LBP (NLBP) will allow determination of the cause of LBP and frequencies of proprioception deficit.

Postural sway of NSLBP during local frequency vibrations of 30,60 and $240 \mathrm{~Hz}$ has been compared with that of NLBP in our previous study. From the result, we found that not only does NSLBP involve the functional impairment of muscle spindles that other groups reported [11-13] but also Vater-Pacini corpuscles were functionally declined because of LBP $[15,16]$. However, in previous studies, only one frequency of vibration stimulation was applied within the range of response frequencies for each proprioceptor $[11-13,15,16]$. In addition, other studies investigating muscle spindle response to multiple vibration frequencies reported different results. A previous study using $90 \mathrm{~Hz}$ vibration frequency suggested that the weighting of proprioceptive feedback from lumbar muscle spindles did not differ between NSLBP and NLBP [17]. On the other hand, another previous study using $60 \mathrm{~Hz}$ vibration frequency suggested that LBP patients rely less on proprioceptive feedback from lumbar muscle spindles [18]. From these results, it is implied that the postural response induced by mechanical vibration varies with the applied vibration frequency even in one proprioceptor. Also, it is assumed that there are individual differences in response frequencies of proprioceptors. To the best of our knowledge, a protocol for measuring postural control while applying local vibration stimulation in the entire response frequency band has not been developed. Thus, we considered that the inspection device and a protocol for evaluating postural response in the range of the entire response frequencies of the proprioceptors are necessary.

Therefore, we developed a new experimental protocol to measure the center of pressure (CoP) of an individual on a gravicorder during local mechanical vibration with sweep frequencies, using our developed variable frequency vibratory stimulation device. This method can be analyzed by applying a vibration stimulus once in the entire frequency range covering the response frequencies of each proprioceptor. For this reason, there is a possibility that this method can identify proprioceptors related to NSLBP and the functionally declined frequency bands of proprioceptors. In addition, the method might be capable of more detailed analyses because the vibration is stimulated with the entire frequency range covering the response frequencies of each proprioceptor. Furthermore, this method is considered to be the shortest for measuring all the response frequency bands of the three proprioceptors. The physical burden on subjects during measurement can also be reduced because the measurement time can be shortened by about $4 \mathrm{~min}$ and $30 \mathrm{~s}$ compared with the time required for the conventional method with a constant frequency. Our final goal is to develop an inspection device for evaluating the functional decline in proprioceptors. In this study, we aimed to investigate the relationship between proprioception and LBP. Thus, postural sway response in NSLBP and NLBP was measured with our new sweep frequency method and compared with the responses obtained from past studies using the method of constant frequency.

\section{Materials and Methods}

\subsection{Subjects}

This study was carried out during a period of 30 months (August 2016-July 2019) in tandem with general clinical practice. Written informed consent was obtained from all participants before 
inclusion in the study. All investigations were conducted according to the principles expressed in the Declaration of Helsinki. The Ethics Committee of the National Center for Geriatrics and Gerontology approved the study (IRB approval number: 586).

Sixty-three elderly individuals (age $\geqq 65$ years: age range $65-90$; sex ratio: 36 women/27 men) were recruited, including 35 patients ( 22 women, 13 men) with NSLBP which lasted over 3 months who visited the National Center for Geriatric and Gerontology for orthopedic treatment. Among the recruited individuals, 28 individuals with NLBP (14 women and 14 men) acted as controls with no history of disabling NSLBP which is defined as LBP when the pathoanatomical cause of the pain cannot be determined [19]. Patients with the following characteristics were excluded-paralysis, astasia, spinal cord tumor, spinal infection and history of spinal surgery. Demographics of all individuals are presented in Table 1.

Table 1. Demographic characteristics and functional outcome of the patients.

\begin{tabular}{cccc}
\hline Variables & NLBP $(\boldsymbol{n}=\mathbf{2 8})$ & NSLBP $(\boldsymbol{n}=\mathbf{3 5})$ & $\boldsymbol{p}$ \\
\hline Age, years & $75.9 \pm 5.4$ & $75.1 \pm 5.2$ & 0.552 \\
Height, $\mathrm{cm}$ & $154.8 \pm 7.7$ & $153.2 \pm 7.3$ & 0.410 \\
Weight, $\mathrm{kg}$ & $59.8 \pm 11.4$ & $57.7 \pm 10.5$ & 0.609 \\
BMI, $\mathrm{kg} / \mathrm{m}^{2}$ & $24.8 \pm 3.3$ & $24.5 \pm 3.7$ & 0.774 \\
VAS, cm & $2.4 \pm 2.5$ & $6.2 \pm 2.4$ & $<0.001$ \\
RDQ (score) & $9.1 \pm 5.8$ & $11.8 \pm 4.0$ & $<0.05$ \\
\hline
\end{tabular}

BMI: body mass index; NLBP: non-low back pain; NSLBP: non-specific low back pain; VAS: visual analog scale; RDQ: Roland-Morris disability questionnaire; SD: standard deviation. Data are presented as the mean $\pm \mathrm{SD}$. All $p$ values were generated using the independent $t$-test, Mann-Whitney $u$ test.

\subsection{Device}

Figure $1 \mathrm{a}, \mathrm{b}$ shows an inspection system for evaluating the functional decline in proprioceptors. The system consists of a PC, an amplifier, four vibrators (NSW1-205-8A, Aurasound, Inc., CA, USA), three hook-and-loop fasteners and a Wii Balance Board (Nintendo Co., Ltd., Kyoto, Japan). Two hook-and-loop fasteners with one vibrator were used for the left and right lower legs and one hook-and-loop fastener with two vibrators was used for the left and right parts of the trunk.

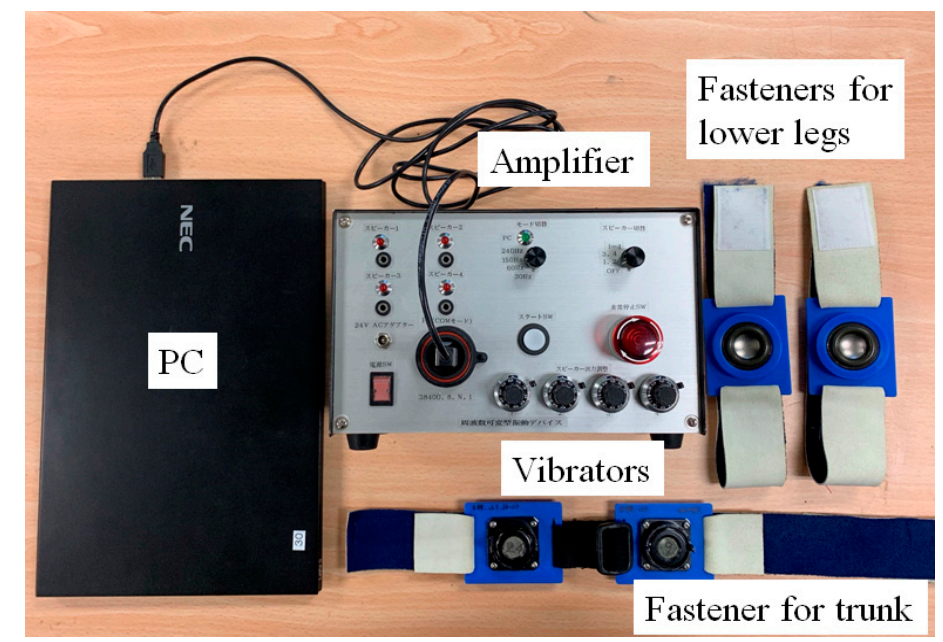

(a)

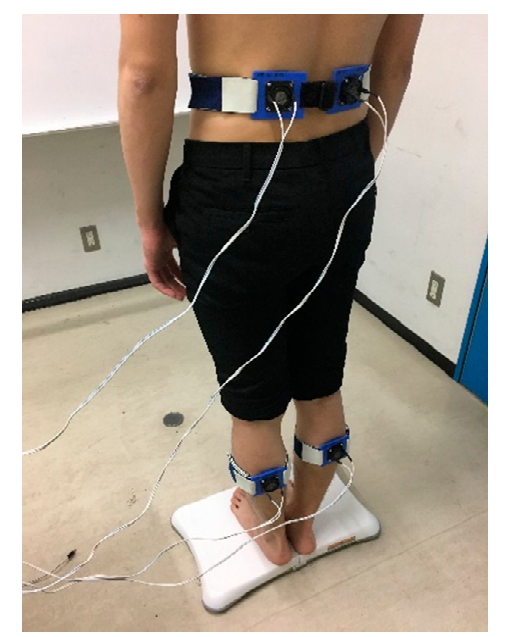

(b)

Figure 1. Inspection system for proprioceptive function: (a) Variable frequency vibratory stimulation device; and (b) experimental setup for a participant.

Vibration signals were generated using the PC. Then, vibrations were output from the vibrators as a mechanical vibration stimulus through the amplifier. In other previous studies, the mechanical 
vibration stimulus with an amplitude of 0.4 to $1.0 \mathrm{~mm}$ was used as the proprioceptive input $[10,11,13]$. Also, the maximum amplitude of our developed device which can be output without distortion of mechanical vibration at each frequency from 20 to $300 \mathrm{~Hz}$ is $0.8 \mathrm{~mm}$. Thus, the amplitude of the vibration was selected at $0.8 \mathrm{~mm}$ of a sinusoidal wave. Vibrators were attached to the hook-and-loop fasteners using holders and fixed to the muscle belly of the gastrocnemius (GS) and the lumbar multifidus (LM) muscles. The contact pressure to the body locations of the vibrators was regulated by changing the length of the hook-and-loop fasteners according to the circumference of the fixed body locations. A Wii Balance Board was used as a gravicorder. The CoP was measured instead of the center of gravity. A Wii Balance Board has been reported as a reliable and valid tool for assessing standing balance [20]. It can acquire the time series data of CoP coordinates with a sampling frequency of $100 \mathrm{~Hz}$. Moreover, it is possible to save the CoP as CSV data by running the self-made CoP data collection software on a PC connected with the Wii Balance Board via Bluetooth.

\subsection{Experimental Method}

The CoP in postural sway while the participant stood barefoot on the Wii Balance Board with his/her eyes closed and feet together was measured. Participants were instructed to remain still and relaxed in the standing position with their arms hanging loosely at their sides. In order to prevent injuries due to falls during measurements, two researchers stood on both sides of a subject with eyes closed. They paid attention to whether or not the subject was likely to fall and gave support if the subject was falling. As the vibration frequency, a sweep frequency (Figure 2) was used. The vibration was continuously changed from $27 \mathrm{~Hz}$ to $272 \mathrm{~Hz}$ (frequency ascend mode) or $272 \mathrm{~Hz}$ to $27 \mathrm{~Hz}$ (frequency descend mode) for $60 \mathrm{~s}$, thus covering the response frequency ranges of the three proprioceptors. The relationships of sweep frequency between frequency $f(t)$ and measurement time $t$ are shown in Equations (1) and (2). The coefficient $a$ was determined so that the frequencies at $t=15 \mathrm{~s}$ and $75 \mathrm{~s}$ were set to $27 \mathrm{~Hz}$ and $272 \mathrm{~Hz}$ in the frequency ascend mode, respectively. Thus, the coefficient $a$ was found to be 0.03851 . Furthermore, the frequency descend mode had the same frequencies at $t=15 \mathrm{~s}$ and $75 \mathrm{~s}$ ( $272 \mathrm{~Hz}$ and $27 \mathrm{~Hz}$, respectively).

$$
\begin{gathered}
f(t)=\left\{\begin{array}{c}
0(0 \leq t \leq 15) \\
27 e^{a(t-15)}(15 \leq t \leq 75)
\end{array} \quad\right. \text { (frequency ascend mode) } \\
f(t)=\left\{\begin{array}{c}
0(0 \leq t \leq 15) \\
27 e^{a(75-t)}(15 \leq t \leq 75)
\end{array}\right. \text { (frequency descend mode) }
\end{gathered}
$$

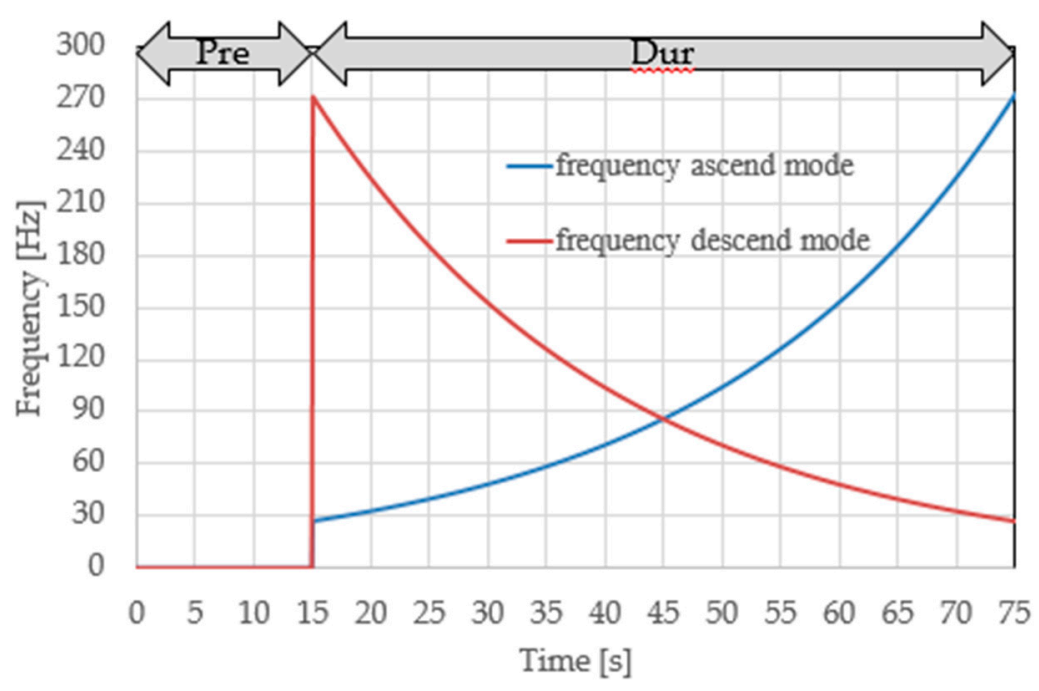

Figure 2. Sweep frequency of vibratory stimulation. 
The measurement procedure consisted of two conditions that provided vibration stimulus-GS and LM. The time needed for one condition was $75 \mathrm{~s}$ and was divided into two sections. The first $15 \mathrm{~s}$ and last $60 \mathrm{~s}$ sections are referred to as Pre- and Dur-sections, respectively. In the Pre-section, a participant closed his/her eyes. Then, in the Dur-section, the vibratory stimulation was applied to the GS or LM of the participant with his/her eyes closed. The ascend or descend sweep frequency mode was randomly determined for each subject. A rest interval of $60 \mathrm{~s}$ was kept between the conditions. During this time, each participant sat in a chair and remained at rest.

\subsection{Data Analysis Method}

The postural control performance was evaluated from the CoP data while vibration stimulation was applied. Furthermore, the CoP data divided the Dur-section into three evaluation sections $\left(\mathrm{ES}_{\mathrm{i}}\right.$, $\mathrm{i}=1,2,3$ ) determined according to the frequency of vibration stimulation and the response frequencies of the proprioceptors. Table 2 lists the names of each ES, the frequency of the vibration stimulus and the corresponding proprioceptors.

Table 2. Lists of names of each ES, frequency of vibration stimulus and corresponding proprioceptors.

\begin{tabular}{ccc}
\hline ES $_{\mathbf{i}}$ & Frequency $[\mathrm{Hz}]$ & Corresponding Proprioceptors \\
\hline $\mathrm{ES}_{1}$ & $30-43$ & Meissner corpuscle \\
$\mathrm{ES}_{2}$ & $49-71$ & Muscle spindles \\
$\mathrm{ES}_{3}$ & $168-247$ & Vater-Pacini corpuscle \\
\hline
\end{tabular}

ES: Evaluation section.

Because the previous studies showed that a relationship existed between CoP displacement in an anteroposterior direction and the response of proprioceptors by vibration stimulus, the CoP displacement in the anteroposterior direction was only considered [10]. Previous studies used the relative proprioceptive weighting ratio (RPW) and the root mean square (RMS) of GS and LM as evaluation indexes to evaluate the proprioceptive predominance of body location and the magnitude of $\mathrm{CoP}$ in an anteroposterior direction $[11,15,18]$. The evaluation indexes applicable to the sweep frequency method were introduced as follows.

To obtain information regarding the proprioceptive predominance of body locations, modified RPW was calculated using the following equation.

$$
R P W^{i}=\frac{R M S_{G S}^{i}}{R M S_{G S}^{i}+R M S_{L M}^{i}} \times 100[\%] \quad(i=1,2,3)
$$

where the subscript $i$ is used to distinguish the ESs. The higher is the value of $R P W^{i}$ from $50 \%$ to $100 \%$, the higher is the reliability of GS input. On the other hand, the lower is the value of $R P W^{i}$ from $50 \%$ to $0 \%$, the higher is the reliability of LM input $[11,15] . R M S_{*}^{i}$ means the effective value of the magnitude of CoP in an anteroposterior direction. In each ES in the Dur-section, the larger the RMS, the higher the amount of transition of postural sway in the anteroposterior direction compared to those in the Pre-section. $R M S_{*}^{i}$ was calculated using the following equations.

$$
\begin{aligned}
R M S_{*}^{1} & =\sqrt{\frac{1}{N} \sum_{n=n_{1}}^{n_{2}}\left\{Y_{\operatorname{Dur}(*)}(n)-\bar{Y}_{\text {pre }(*)}\right\}^{2}} \\
R M S_{*}^{2} & =\sqrt{\frac{1}{N} \sum_{n=n_{3}}^{n_{4}}\left\{Y_{\operatorname{Dur}(*)}(n)-\bar{Y}_{\operatorname{pre}(*)}\right\}^{2}}
\end{aligned}
$$




$$
R M S_{*}^{3}=\sqrt{\frac{1}{N} \sum_{n=n_{5}}^{n_{6}}\left\{Y_{\operatorname{Dur}(*)}(n)-\bar{Y}_{\text {pre }(*)}\right\}^{2}}
$$

where $N$ is the total sampling number of each ES; $n$ is the number of data series; $Y_{\operatorname{Dur}(*)}(n)$ is the CoP in the anteroposterior direction in the Dur-section; $\bar{Y}_{\text {pre }(*)}$ is the mean value of $\mathrm{CoP}$ in the anteroposterior direction in the Pre-section; the superscript " $*$ " is used to distinguish stimulation body locations, namely, the GS or LM; and subscript numbers are used to distinguish the ESs. In this case, $N$ was equal to 1000 because all ESs were analyzed in $10 \mathrm{~s}$ and the sampling frequency was $100 \mathrm{~Hz}$. Table 3 shows the numbers of data series corresponding to the start frequency and the end frequency of $\mathrm{ES}_{\mathrm{i}}$ in the case of frequency ascend mode and descend mode. In each of the ascend and descend modes, the numbers of sampling series $n$ corresponding to measurement time $t$ were calculated by Equations (1) and (2) in order to obtain the start and end frequencies of each ES, respectively, as shown in Table 2. To correct the $\mathrm{CoP}$ in the Dur-section based on the CoP in the Pre-section, the CoP in the Dur-section was subtracted from the mean value of the CoP in the Pre-section. These values were calculated using MATLAB (MathWorks, Inc., Natick, MA, USA).

Table 3. Numbers of data series corresponding to the start and end frequencies of $\mathrm{ES}_{\mathrm{i}}$.

\begin{tabular}{ccccc}
\hline \multirow{2}{*}{ SS $_{\mathbf{i}}$} & $\begin{array}{c}\text { Number of } \\
\text { Data Series }\end{array}$ & $\begin{array}{c}\text { Frequency } \\
\text { Ascend Mode }\end{array}$ & $\begin{array}{c}\text { Frequency } \\
\text { Descend Mode }\end{array}$ & $\begin{array}{c}\text { Corresponding } \\
\text { Frequency [Hz] }\end{array}$ \\
\hline \multirow{2}{*}{$\mathrm{ES}_{1}$} & $n_{1}$ & 1750 & 7250 & 30 \\
& $n_{2}$ & 2750 & 6250 & 43 \\
\hline \multirow{2}{*}{$\mathrm{ES}_{2}$} & $n_{3}$ & 3000 & 6000 & 49 \\
& $n_{4}$ & 4000 & 5000 & 71 \\
\hline \multirow{2}{*}{$\mathrm{ES}_{3}$} & $n_{5}$ & 6250 & 2750 & 247 \\
\hline
\end{tabular}

ES: evaluation section; $n$ : the number of data series; The subscript numbers of ES are indicated as follows: "1: Meissner corpuscle; from 30 to $43 \mathrm{~Hz}$; 2: muscle spindles; from 49 to $71 \mathrm{~Hz}$, 3: Vater-Pacini corpuscle; from 168 to $247 \mathrm{~Hz} . "$

\subsection{Statistical Analysis}

All analyses were performed using IBM SPSS statistical software (Version 25.0; IBM Corp., Armonk, NY, USA). The frequency ascend and descend modes groups were combined into a population of $R P W^{i}$ and $R M S_{*}^{i}$ for statistical analysis to eliminate the order effects due to vibration application. Data were expressed as mean values and standard deviations for the NLBP and NSLBP. First, these data were analyzed to investigate whether they were normally distributed using the Shapiro-Wilk test. Variable data of $R P W^{i}$ were compared between NLBP and NSLBP using the independent t-test if both the compared data were normally distributed. In statistical analysis, $p<0.05\left(^{*}\right)$ were considered statistically significant. In addition, a two-way mixed analysis of variance (ANOVA) was performed with RMS as the dependent variable. The independent variables were group (NSLBP group or NLBP group) and the stimulated body locations (GS or LM). Where significant interaction effects were identified, paired $t$-tests and univariate analysis with Bonferroni correction were conducted.

\section{Results}

\subsection{Proprioceptive Postural Control}

Table 4 shows the results of $R P W^{i}$. For each evaluation index, the mean values and SDs analyzed in the 3 sections are shown. The RPW values of NSLBP were smaller than 50\%. NSLBP had a significantly smaller RPW compared with NLBP in Vater-Pacini corpuscles $(p<0.05)$. Meanwhile, there were no significant differences in the RPW values of Meissner corpuscles and muscle spindle between NSLBP and NLBP groups (Table 4). 
Table 4. Mean values of $R P W^{\mathrm{i}}$ with standard deviations.

\begin{tabular}{cccc}
\hline Variable & NLBP & NSLBP & $p$ \\
\hline$R P W^{1}[\%]$ & $52.2 \pm 13.2$ & $48.2 \pm 9.3$ & 0.184 \\
$R P W^{2}[\%]$ & $53.8 \pm 11.8$ & $49.1 \pm 10.2$ & 0.092 \\
$R P W^{3}[\%]$ & $53.3 \pm 12.5$ & $46.4 \pm 9.8$ & $<0.05$
\end{tabular}

NLBP: non-low back pain; NSLBP: non-specific low back pain; RPW: relative proprioceptive weighting ratio. Data are presented as the mean \pm standard deviation or $n[\%]$. All $p$ values were generated using an independent $t$-test. The superscript numbers indicated the following: "1: Meissner corpuscle; from 30 to $43 \mathrm{~Hz}$; 2: muscle spindles; from 49 to $71 \mathrm{~Hz}$, 3: Vater-Pacini corpuscle; from 168 to $247 \mathrm{~Hz} . "$

\subsection{Postural Stability}

Table 5 shows a result of a two-way mixed ANOVA in $R M S_{*}^{i}$. There was no significant interaction between the NLBP and NSLPB group and the stimulated body locations in the Meissner corpuscle and muscle spindle. In contrast, the interaction between the NLBP and NSLPB groups and the stimulated body locations in Vater-Pacini corpuscles were significantly observed $(p<0.05)$.

Figure 3 depicts the results of RMS in Vater-Pacini corpuscle. Compared with NLBP group, univariate analysis with Bonferroni correction showed that the NSLBP group exhibited significantly higher RMS values at LM in the Vater-Pacini corpuscle $(\mathrm{F}(1,61)=4.817, p<0.05)$, whereas there was no significant difference in the RMS values at GS in the Vater-Pacini corpuscle $(F(1,61)=0.115, p=0.735)$. In addition, the paired $t$-test showed that there was a tendency of significantly higher RMS values at LM than at GS in the NSLBP group $(p=0.055)$. There was no significant difference between GS and LM in the NLBP group $(p=0.189)$.

Table 5. Results of $R M S_{*}^{i}$ and two-way mixed analysis of variance.

\begin{tabular}{ccccc}
\hline Variable & NLBP & NSLBP & F(1, 61) & $p$ \\
\hline$R M S_{G S}^{1}[\mathrm{~mm}]$ & $10.7 \pm 7.8$ & $8.9 \pm 4.3$ & 2.874 & 0.095 \\
$R M S_{L M}^{1}[\mathrm{~mm}]$ & $8.7 \pm 4.0$ & $9.5 \pm 4.3$ & & \\
\hline$R M S_{G S}^{2}[\mathrm{~mm}]$ & $10.9 \pm 7.4$ & $9.6 \pm 4.6$ & 2.624 & 0.110 \\
$R M S_{L M}^{2}[\mathrm{~mm}]$ & $8.8 \pm 4.3$ & $9.7 \pm 4.5$ & & \\
\hline$R M S_{G S}^{3}[\mathrm{~mm}]$ & $9.6 \pm 4.0$ & $9.2 \pm 3.9$ & 5.255 & $<0.05$ \\
$R M S_{L M}^{3}[\mathrm{~mm}]$ & $8.4 \pm 3.6$ & $10.8 \pm 4.9$ & & \\
\hline
\end{tabular}

NLBP: non-low back pain; NSLBP: non-specific low back pain; RMS: root mean square of the center of pressure data. Data are presented as the mean \pm standard deviation or $n[\%]$. All $\mathrm{F}$ value and $p$ values were generated using the two-way mixed analysis of variance. Independent variables: the NLBP and NSLPB group and the stimulated body locations. The subscripts and superscript numbers indicated the following: "GS: gastrocnemius; LM: lumbar multifidus; 1: Meissner corpuscle; from 30 to $43 \mathrm{~Hz}$; 2: muscle spindles; from 49 to $71 \mathrm{~Hz}$, 3: Vater-Pacini corpuscle; from 168 to $247 \mathrm{~Hz} . "$

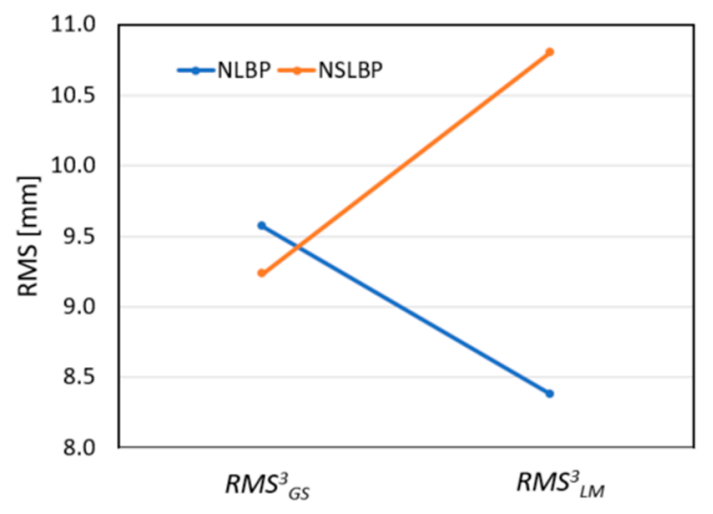

Figure 3. Result of RMS in Vater-Pacini corpuscle. RMS indicates root mean square of the center of pressure data; GS: gastrocnemius; LM: lumbar multifidus. 


\section{Discussion}

To our knowledge, this is the first study to evaluate the postural response when vibration was applied in the entire response frequency range of proprioceptors using sweep frequency. Our main finding was that NSLBP relied more on LM input in the Vater-Pacini corpuscles during vibratory stimulus with a sweep frequency of $168-247 \mathrm{~Hz}$ to the lower leg in the standing position.

Past studies have already demonstrated that elderly patients with LBP relied on trunk strategies (Vater-Pacini corpuscles) for postural control during vibratory stimulation $[15,16]$. Compared with NLBP, lower RPW values in NSLBP during vibratory stimulations of 168 to $247 \mathrm{~Hz}$ indicate that NSLBP relies on trunk-muscle proprioceptive inputs. Moreover, the reduction in lower leg sensitivity at the Vater-Pacini corpuscles in the NSLBP group agrees well with the result of previous studies which reported that the NSLBP group decreased its reliance on proprioceptive signals from the lower leg during standing at $240 \mathrm{~Hz}$ of constant vibratory stimulus [15,16]. However, statistically significant differences were not observed between the NLBP and NSLBP groups in the Meissner corpuscles and muscle spindle. The decreased reliability on LM inputs in the Meissner corpuscles and muscle spindle may not have been recognized in the NSLBP group because this study was performed under different conditions from measurement methods and subjects in the previous studies [10-12,21-24]. In addition, the differences in age and LBP degree may have affected even the same cases of disease $[15,16]$. Therefore, the NSLBP group may depend more on the Vater-Pacini corpuscles of their trunk than on the Meissner corpuscles and muscle spindle.

Second, the resulting RMS values were considered. A significant interaction between the NLBP and NSLPB groups and the stimulated body location in Vater-Pacini corpuscles was confirmed. From Figure 3, the NSLBP group exhibited higher RMS values when vibration in the frequency band of the Vater-Pacini corpuscles was applied to LM. This result supports that the NSLBP group relied more on LM input in the Vater-Pacini corpuscles for postural control. These results agree well with those of previous studies where significant differences in postural sways were found between elderly individuals and healthy young adults during quiet standing conditions [25]. Moreover, a decreasing trend in the proprioceptive function in lower leg was observed in the NSLBP group. These findings clearly show the differences in postural control strategies of proprioceptive inputs with respect to NSLBP, suggesting that LBP may influence the postural control strategy of proprioceptive inputs derived from the Vater-Pacini corpuscles of the LM.

For the above reasons, we confirmed that the function of the Vater-Pacini corpuscles was decreased in relation to LBP using the sweep frequency method. Ito et al. reported that patients with NSLBP may have even greater postural instability because the sensitivity of GS at $240 \mathrm{~Hz}$ continues to decline [15]. Thus, the reduction possibly causes postural instability because the NSLBP group cannot switch to a more appropriate proprioceptive postural strategy. In addition, the effect of RPW on postural control under proprioceptive stimulation with the response frequency of Vater-Pacini corpuscles may be a good indicator of evaluating the degree of LBP under the sweep frequency method, same as the constant frequency method used in our previous study [15]. These findings could be used to diagnose NSLBP due to the reduced function of proprioceptors. The advantages of using the sweep frequency are as follows. Firstly, the sweep frequency method may eliminate individual differences in the response frequency of proprioceptors. Secondly, the resolution is high because the vibration is applied to the entire frequency band of the response frequency of each proprioceptor. In the future, it may be possible to conduct more detailed proprioceptive examination by analyzing finer frequency bands. Additionally, there is a possibility that the inspection using this method can identify the detailed frequency band of proprioceptors related to NSLBP. Thirdly, the examination time could be shortened. The measurement time of the sweep frequency method can be shortened by $4 \mathrm{~min}$ and $30 \mathrm{~s}$ compared with the time required for the constant frequency method used in our previous study. Thus, this method can reduce the burden on patients. Therefore, the method using sweep frequency could replace the conventional constant frequency method because it can measure the entire frequency band of proprioceptors in a short time. 
Nevertheless, this study has a limitation in that only elderly persons with lumbar spondylosis were surveyed. Therefore, additional studies with healthy older persons and those with more severe disability must be conducted. Finally, the evaluation of the entire range of response frequencies of proprioceptors using sweep frequency could benefit from future studies investigating more optimal stimulation of response frequencies.

\section{Conclusions}

In this study, we aimed to investigate the functional decline in proprioceptors related to LBP by evaluating the entire range of response frequencies of proprioceptors using sweep frequency. Thus, we developed a variable frequency vibratory stimulation device and measured the CoP of NSLBP and NLBP on a Wii Balance Board during local vibration with sweep frequencies. As a result, compared with the NLBP group, the NSLBP group showed a reduction in lower leg sensitivity at Vater-Pacini corpuscles. Moreover, we believe that the sweep frequency method can replace the conventional constant frequency method because it can measure the entire frequency band of proprioceptors in a short time. Our future work will include investigating the treatment effect of vibration with the frequency evaluated by this device.

Author Contributions: Conceptualization, Y.M. and Y.S.; methodology, Y.M.; software, R.N., Y.I. and K.Y.; validation, T.I.; formal analysis, R.N.; investigation, R.N., Y.I., T.I. and K.Y.; resources, Y.M. and Y.S.; data curation, R.N. and Y.I.; writing —original draft preparation, R.N.; writing—review and editing, Y.I., Y.M., T.I., K.Y. and Y.S.; visualization, R.N.; supervision, Y.M.; project administration, Y.M. and Y.S.; funding acquisition, Y.S.

Funding: This research was funded by Geriatrics and Gerontology research and development cost, grant number 29-6. The APC was funded by Geriatrics and Gerontology research and development cost.

Acknowledgments: The authors thank the staff in the Department of Orthopedic Surgery and the National Center for Geriatric and Gerontology for their help in participant recruitment. This study was supported in part by a grant from the Geriatrics and Gerontology research and development cost.

Conflicts of Interest: The authors declare no conflicts of interest.

\section{References}

1. Hoy, D.; March, L.; Brooks, P.; Blyth, F.; Woolf, A.; Bain, C.; Williams, G.; Smith, E.; Vos, T.; Barendregt, J.; et al. Global burden of low back pain: Estimates from the Global Burden of Disease 2010 study. Ann. Rheum. Dis. 2014, 76, 968-974. [CrossRef] [PubMed]

2. Andrea, R.; Cholewicki, J.; Polzhofer, G.K.; Greene, H.S. Impaired postural control of the lumbar spine is associated with delayed muscle response times in patients with chronic idiopathic low back pain. Spine 2001, 26, 724-730. [CrossRef]

3. Henry, S.; Hitt, J.; Jones, S.; Bunn, J. Decreased limits of stability in response to postural perturbations in subjects with low back pain. Clin. Biomech. 2006, 21, 881-892. [CrossRef] [PubMed]

4. Willigenburg, N.W.; Kingma, I.; van Dieën, J.H. How is precision regulated in maintaining trunk posture? Exp. Brain Res. 2010, 203, 39-49. [CrossRef] [PubMed]

5. Sean, C.; Tim, K.; John, J.J. Modeling the dynamics of sensory reweighting. Biol. Cybern. 2006, 95, 123-134. [CrossRef]

6. Johansson, R.; Magnusson, M. Human postural dynamics. Crit. Rev. Biomed. Eng. 1991, 18, $413-437$.

7. Willigenburg, N.W.; Kingma, I.; Hoozemans, M.J.; van Dieën, J.H. Precision control of trunk movement in low back pain patients. Hum. Mov. Sci. 2013, 32, 228-239. [CrossRef]

8. Bloem, B.R.; Allum, J.M.; Carpenter, M.G.; Honegger, F. Is lower leg proprioception essential for triggering human automatic postural responses? Exp. Brain Res. 2000, 130, 375-391. [CrossRef]

9. Kuroki, S.; Hagura, N.; Nishida, S.; Haggard, P.; Watanabe, J. Sanshool on The Fingertip Interferes with Vibration Detection in a Rapidly-Adapting (RA) Tactile Channel. PLoS ONE 2016, 11. [CrossRef]

10. Pyykko, I.; Jantti, P.; Aalto, H. Postural control in elderly subjects. Age Ageing 1990, 19, 215-221. [CrossRef]

11. Brumagne, S.; Janssens, L.; Janssens, E.; Goddyn, L. Altered postural control in anticipation of postural instability in persons with recurrent low back pain. Gait Posture 2008, 28, 657-662. [CrossRef] [PubMed] 
12. Goossens, N.; Janssens, L.; Pijnenburg, M.; Severijns, P.; Van, R.C.; Meugens, P.; Brumagne, S. Test-Retest Reliability and Concurrent Validity of an fMRI-Compatible Pneumatic Vibrator to Stimulate Muscle Proprioceptors. Multisens. Res. 2016, 29, 465-492. [CrossRef] [PubMed]

13. Capicíková, N.; Rocchi, L.; Hlavacka, F.; Chiari, L.; Cappello, A. Human postural response to lower leg muscle vibration of different duration. Physiol. Res. 2006, 55, 129-134.

14. Nima, T.; Hossein, E.; Marco, M.; Jane, M. Proprioceptive impairments in high fall risk older adults: The effect of mechanical calf vibration on postural balance. BioMed. Eng. Online 2018, 17, 51. [CrossRef]

15. Ito, T.; Sakai, Y.; Morita, Y.; Yamazaki, K.; Igarashi, K.; Nishio, R.; Sato, N. Proprioceptive Weighting Ratio for Balance Control in Static Standing Is Reduced in Elderly Patients with Non-Specific Low Back Pain. Spine 2018, 43, 1704-1709. [CrossRef]

16. Ito, T.; Sakai, Y.; Yamazaki, K.; Igarashi, K.; Sato, N.; Yokoyama, K.; Morita, Y. Proprioceptive change impairs balance control in older patients with low back pain. J. Phys. Ther. Sci. 2017, 29, 1788-1792. [CrossRef]

17. Willigenburg, N.W.; Kingma, I.; van Dieën, J.H. Precision control of an upright trunk posture in low back pain patients. Clin. Biomech. 2012, 27, 866-871. [CrossRef]

18. Brumagne, S.; Cordo, P.; Verschueren, S. Proprioceptive weighting changes in persons with low back pain and elderly persons during upright standing. Neurosci. Lett. 2004, 366, 63-66. [CrossRef]

19. Chris, M.; Martin, U.; Rachelle, B. Non-specific low back pain. Lancet 2017, 389, 736-747. [CrossRef]

20. Clark, R.A.; Mentiplay, B.F.; Pua, Y.H.; Bower, K.J. Reliability and validity of the Wii Balance Board for assessment of standing balance: A systematic review. Gait Posture 2018, 61, 40-54. [CrossRef]

21. Claeys, K.; Brumagne, S.; Dankaerts, W.; Kiers, H.; Janssens, L. Decreased variability in postural control strategies in young people with non-specific low back pain is associated with altered proprioceptive reweighting. Eur. J. Appl. Physiol. 2011, 111, 115-123. [CrossRef] [PubMed]

22. Brumagne, S.; Janssens, L.; Knapen, S.; Claeys, K.; Suuden-Johanson, E. Persons with recurrent low back pain exhibit a rigid postural control strategy. Eur. Spine J. 2008, 17, 1177-1184. [CrossRef] [PubMed]

23. Mientjes, M.I.; Frank, J.S. Balance in chronic low back pain patients compared to healthy people under various conditions in upright standing. Clin. Biomech. 1999, 14, 710-716. [CrossRef]

24. Ito, T.; Sakai, Y.; Nakamura, E.; Yamazaki, K.; Yamada, A.; Sato, N.; Morita, Y. Relationship between paraspinal muscle cross-sectional area and relative proprioceptive weighting ratio of older persons with lumbar spondylosis. J. Phys. Ther. Sci. 2015, 27, 2247-2251. [CrossRef] [PubMed]

25. Ito, T.; Sakai, Y.; Yamazaki, K.; Nishio, R.; Ito, Y.; Morita, Y. Postural Strategy in Elderly, Middle-Aged, and Young People during Local Vibratory Stimulation for Proprioceptive Inputs. Geriatrics 2018, 3, 93. [CrossRef]

(C) 2019 by the authors. Licensee MDPI, Basel, Switzerland. This article is an open access article distributed under the terms and conditions of the Creative Commons Attribution (CC BY) license (http://creativecommons.org/licenses/by/4.0/). 